\title{
Trigeminal Neuralgia due to an Isolated Cerebral Varix: Case Report
}

\author{
Takuro Inoue $^{1}$ Ayako Shima ${ }^{1}$ Hisao Hirai ${ }^{1}$ Fumio Suzuki ${ }^{1}$ Masayuki Matsuda ${ }^{1}$ \\ ${ }^{1}$ Department of Neurosurgery, Subarukai Kotoh Kinen Hospital, \\ Higashiohmi, Shiga, Japan \\ J Neurol Surg Rep 2014;75:e206-e209.

\begin{abstract}
Address for correspondence Takuro Inoue, MD, PhD, 2-1 Hiramatsucho, Higashiohmi-shi, Shiga 527-0134, Japan

(e-mail: takuro39@gmail.com).
\end{abstract}

\author{
Abstract \\ Keywords \\ - trigeminal neuralgia \\ - developmental \\ venous anomaly \\ - varix \\ - 3D imaging
}

Intracranial isolated varix is a very rare entity. Although it is usually asymptomatic, there are reports on symptomatic cases with hemorrhage or mass effect that mostly relate to arteriovenous fistulas or arteriovenous malformations. We present an extremely rare case of trigeminal neuralgia caused by an isolated varix. A 55-year-old woman had been experiencing right trigeminal neuralgia for 3 years. Computed tomography and magnetic resonance imaging revealed an enhanced mass lesion on the root entry zone of the right trigeminal nerve. Angiograms confirmed the mass was a varix arising on the vein connecting the basal vein of Rosenthal and the superior petrosal vein. Preoperative three-dimensional (3D) imaging clearly depicted the anatomical relation of the varix, adjacent vessels, and trigeminal nerve, which helped plan operative procedures. The varix with its parent vein was successfully transposed from the nerve without sacrificing any veins. Her pain disappeared immediately after the surgery and did not recur during a 30-month follow-up period. The 3D image contributed to making an accurate and safer operative plan especially for this rare case.

\section{Introduction}

Isolated cerebral varices are commonly reported in relation to high-flow draining veins of arteriovenous fistulas, arteriovenous malformations, and venous angiomas. ${ }^{1,2}$ Although they usually appear without neurologic symptoms, there are a few reports of symptomatic cases with hemorrhage or mass effect. $^{3,4}$ To the best of our knowledge, there is no report that an isolated cerebral varix causes trigeminal neuralgia.

\section{Case Report}

A 55-year-old woman had been experiencing lancinating pain in her right cheek while eating, brushing her teeth, and washing her face for 3 years. Without pain, her facial sensory was normal. Magnetic resonance imaging (MRI) (fast imaging employing steady-state acquisition [FIESTA]) showed a mass lesion, $\sim 6 \mathrm{~mm}$ in diameter, compressing the root entry zone (REZ) of the right trigeminal nerve ( $\mathbf{- F i g . 1 A}$ ). The mass was

received

April 9, 2014

accepted

May 13, 2014

published online

August 5, 2014
DOI http://dx.doi.org/ $10.1055 / \mathrm{s}-0034-1383861$. ISSN 2193-6358. almost equally enhanced with adjacent veins on contrastenhanced T1 spoiled gradient recalled (SPGR) MRI (-Fig. 1B). Angiogram revealed the mass was a varix arising from the connecting vein between the basal vein of Rosenthal and the superior petrosal vein ( $\mathbf{F i g} . \mathbf{2 A}-\mathbf{C}$ ). No other vascular anomalies, such as arteriovenous fistulas, arteriovenous anomalies, or venous angiomas, were recognized on the angiogram. A three-dimensional (3D) computed tomography (CT) angiogram also indicated the mass was a varix arising on the connecting vein between the basal vein and the superior petrosal vein (-Fig. 2D). Carbamazepine was initially effective to reduce her pain. However, it became difficult to continue medication because of its side effects. As the pain worsened, microvascular decompression of the trigeminal nerve was considered. Three-dimensional imaging constructed by Leksell GammaPlan (Elekta AB, Stockholm, Sweden) clearly depicted the varix compressing the REZ of the trigeminal nerve (-Fig. 3A). Surgery was undertaken via the retrosigmoid approach. The actual anatomical findings were identical to
License terms

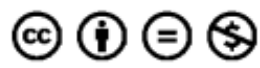

(c) 2014 Georg Thieme Verlag KG Stuttgart · New York 

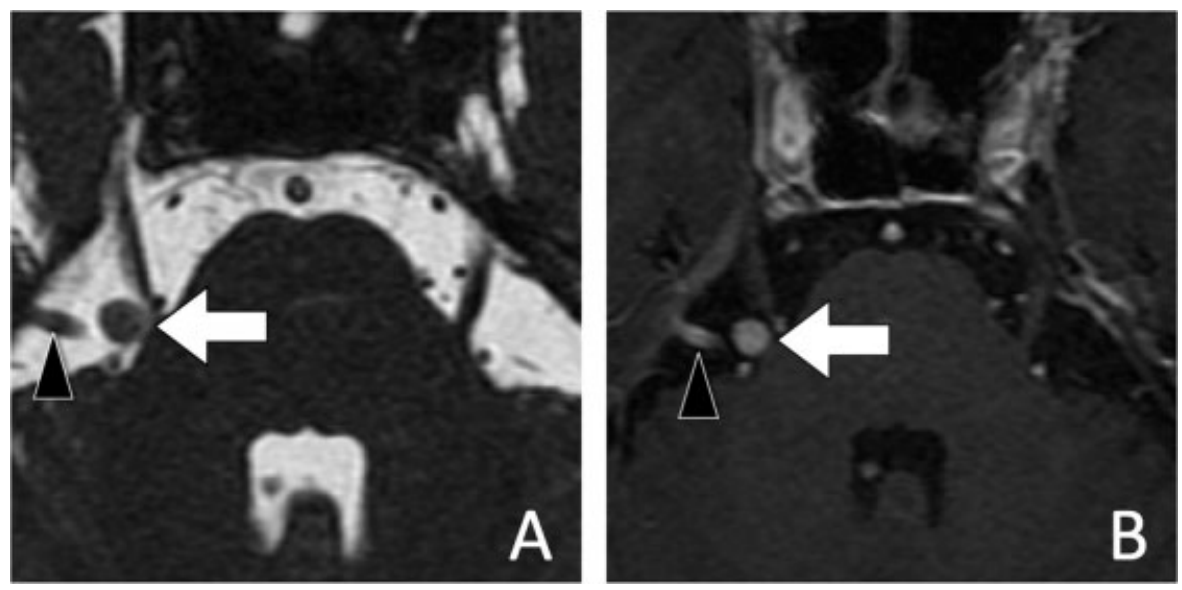

Fig. 1 (A) The axial magnetic resonance imaging (MRI) (fast imaging employing steady-state acquisition) slice at the right trigeminal nerve root shows a mass lesion (white arrow) on the root entry zone of the trigeminal nerve. (B) The contrast enhanced MRI (T1 spoiled gradient recalled) reveals the mass (white arrow) is equally enhanced with surrounding veins (black arrowheads).

the preoperative imaging ( $\mathbf{F i g}$. 3A, B). The superior aspect of the REZ was markedly compressed by the varix, which was considered to be the major cause of the neuralgia (-Fig. $\mathbf{3 C}$ ). The varix was transposed with Teflon felt to the tentorium, together with the connecting vein and the superior petrosal vein without cutting any other veins ( - Fig. 3D). The superior cerebellar artery was also transposed to the tentorium in case it might become a possible cause of trigeminal neuralgia in the future. She was relieved from the pain immediately after the surgery and kept pain free during a 30-month follow-up.
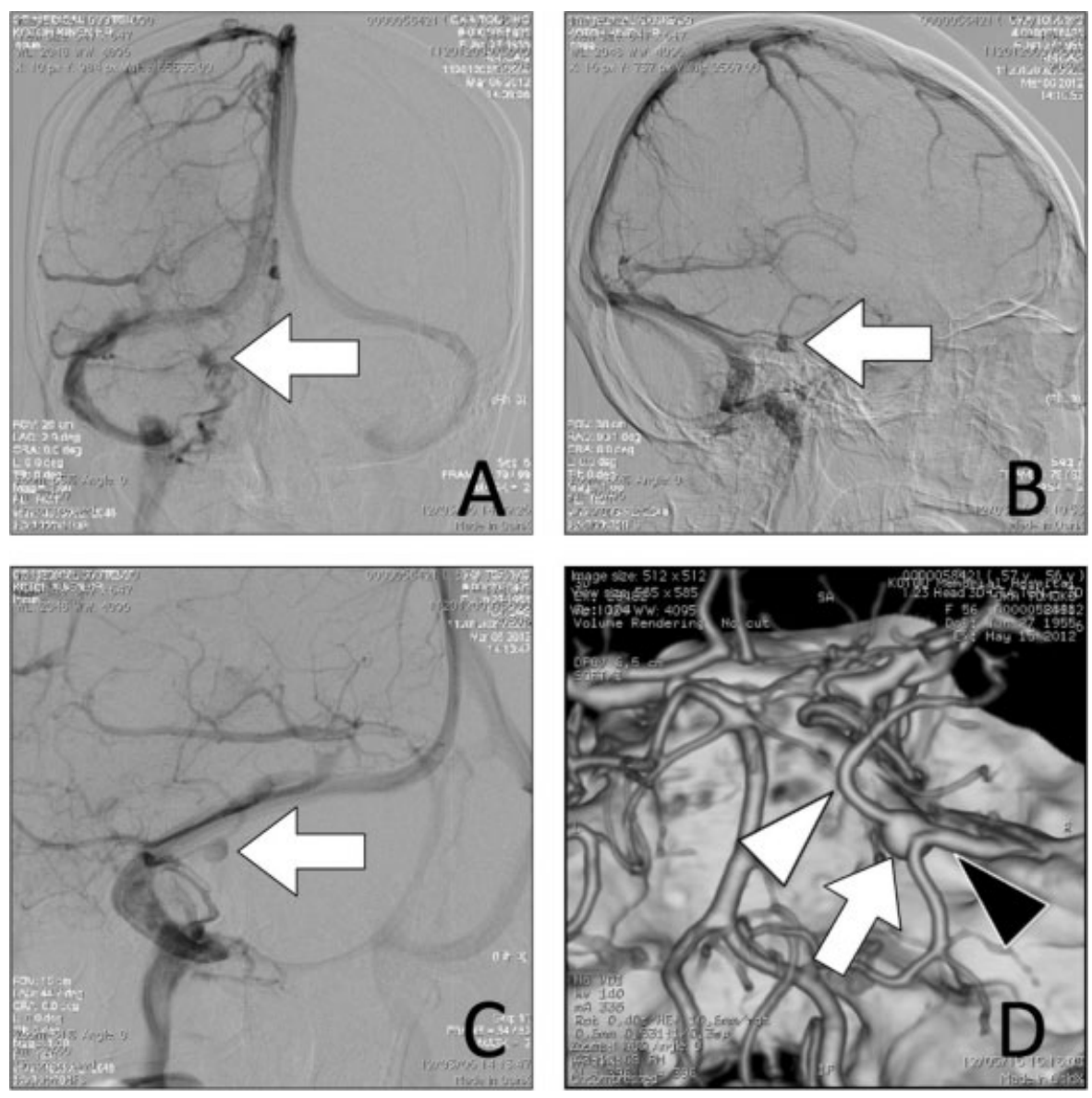

Fig. 2 (A-C) Angiograms show the venous anomaly (white arrows) arises from the vein connecting the basal vein of Rosenthal and the superior petrosal vein. No other abnormality is noted, suggesting the venous anomaly is a varix. (D) Three-dimensional computed tomography shows the varix (white arrow) arises from the vein (white arrowhead), connecting the basal vein to the right superior petrosal vein (black arrowhead). 

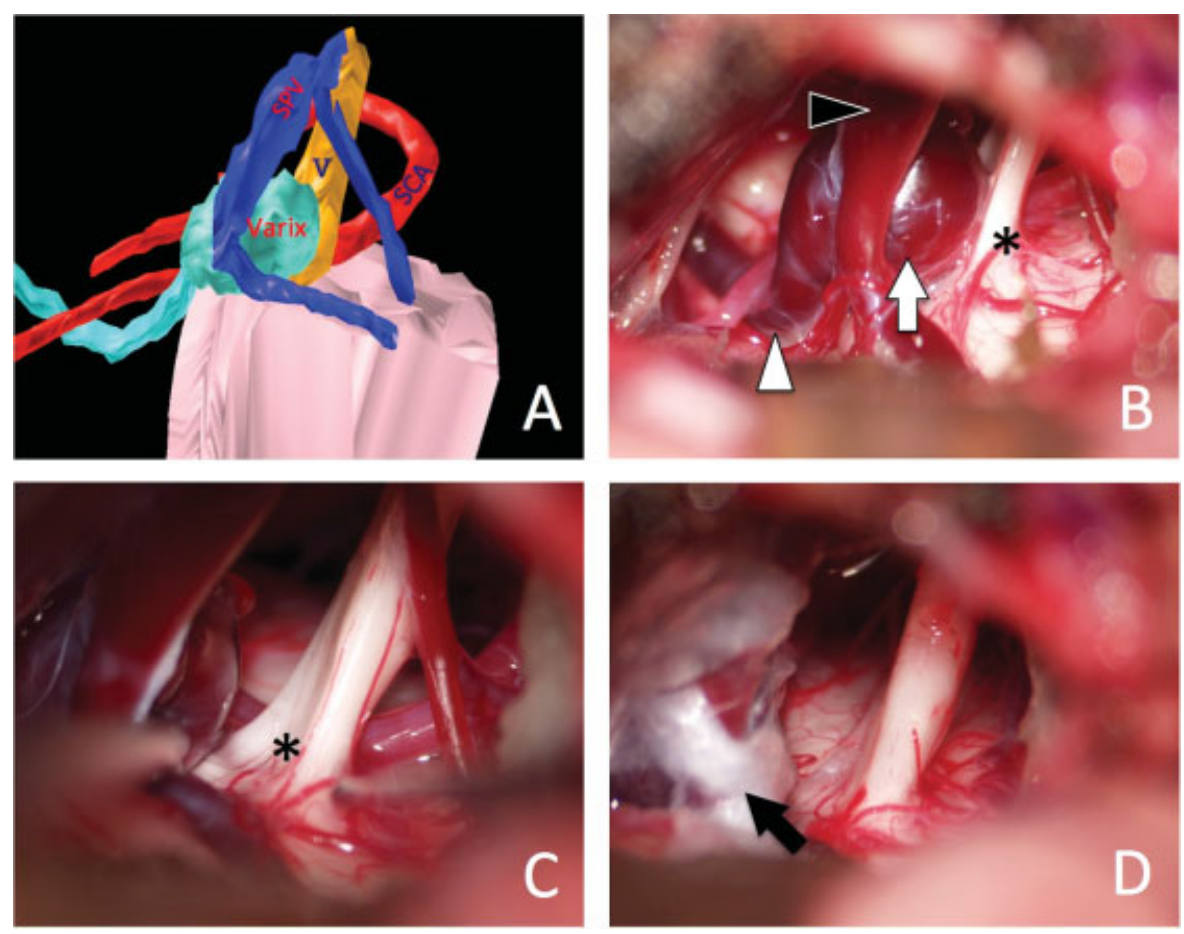

Fig. 3 (A) Preoperative three-dimensional image constructed by GammaPlan. Surgeon's view via the right retrosigmoid approach clearly demonstrates the anatomical relations of the varix, adjacent vessels, and trigeminal nerve. SCA, superior cerebellar artery; SPV, superior petrosal vein; $\mathrm{V}$, trigeminal nerve. (B) Before decompression, the varix (white arrow) arising from the connecting vein (white arrowheads) is compressing the root entry zone (REZ) of the right trigeminal nerve (asterisk). The vein joins into the superior petrosal vein (black arrowhead) just distal to the varix. (C) A marked indentation by the varix is seen on the REZ of the trigeminal nerve (asterisk). (D) The varix and superior petrosal vein are transposed to the tentorium with Teflon felt (black arrow). The superior cerebellar artery is also transposed away from the nerve, just in case.

\section{Discussion}

It is widely accepted that trigeminal neuralgia is caused by neurovascular compression of the trigeminal nerve root. Although it is caused by arterial compression in most cases, venous compression or conflict can be a reason for trigeminal neuralgia. In previous reports, culprit veins were commonly the superior petrosal vein and its tributaries and rarely developmental venous anomalies such as draining veins from venous angiomas. ${ }^{5,6}$

Isolated cerebral varix is a rare entity that usually occurs on a high-flow vein of arteriovenous fistulas or arteriovenous malformations. ${ }^{1,2}$ It is also found in relation to developmental venous anomalies. ${ }^{7,8}$ However, the venous varix is rarely an isolated lesion without any relation to these vascular anomalies. An isolated venous varix is usually asymptomatic and commonly found incidentally. ${ }^{9}$ Therefore, surgical exploration is limited except in rare symptomatic cases such as bleeding or mass effect by its expansion., ${ }^{3,4}$ The present case is such a rare singular isolated varix manifesting a neurologic symptom.

Isolated varix is depicted as an enhanced saccular lesion on CT and signal flow void on MRI. Because this lesion mimics meningioma or cavernoma, ${ }^{9,10}$ angiograms may contribute to establish a diagnosis of isolated varix by showing a focal saccular dilatation on a single vein. Absence of multiple dilated anomalous veins, transparenchymal veins, or dilated draining veins allows distinguishing varices from venous angiomas.
Three-dimensional imaging has been reported to be very helpful for the operative planning of microvascular decompression. $6,11,12$ In patient with trigeminal neuralgia, we use both FIESTA and T1 SPGR contrast-enhanced MRIs. Then we construct 3D imaging using GammaPlan, software for gamma knife surgery, to visualize the exact site of the neurovascular conflict. The surgeon's view enables us to clearly understand the anatomical relation of the nerve and adjacent vessels so we can make an exact plan for decompression before surgery. ${ }^{6,13}$ In the present case, the varix, its related veins, and the site of neurovascular compression were clearly depicted to facilitate understanding of this rare pathologic condition.

Trigeminal neuralgia due to the conflict of veins is rarely reported. In the literature, 5.5 to $16.6 \%$ of trigeminal neuralgia is caused by veins. ${ }^{14}$ It is reported that trigeminal neuralgia caused by veins tends to recur more frequently than by arteries. $^{15,16}$ Hong et al demonstrated a coagulating and cutting technique for decompression when veins adhere to the nerve. ${ }^{5}$ However, the division of the culprit vein in this region carries a risk of brainstem venous infarction. To obtain complete decompression without cutting the culprit veins, careful preoperative planning and dissection of enough length of the veins is required for transposition.

In this case, preoperative 3D imaging clearly indicated the site of neurovascular compression on the superior side of REZ, which allowed us to predict that transposition might be possible by thorough dissection of the superior petrosal vein and its tributaries. Although the superior cerebellar 
artery running on the other side of the nerve could be related to the neuralgia, we believed the marked indentation on the nerve caused by the varix would be the major cause. Thus 3D imaging was useful to plan the operative procedures and contribute to a safer and secure operation.

\section{Conclusion}

Because a cerebral varix is usually asymptomatic, surgical exploration for this entity is quite rare. We presented a rare case of a cerebral varix causing trigeminal neuralgia that was successfully treated by microvascular decompression. Threedimensional imaging is useful for understanding the anatomical relations of veins and trigeminal nerve preoperatively.

\section{References}

1 Chakraborty S, Eldridge P, Nahser HC. Cerebral haemorrhage from a remote varix in the venous outflow of an arteriovenous malformation treated successfully by embolisation. Br J Radiol 2010; 83(991):e129-e134

2 Viñuela F, Drake CG, Fox AJ, Pelz DM. Giant intracranial varices secondary to high-flow arteriovenous fistulae. J Neurosurg 1987; 66(2):198-203

3 Tyson GW, Jane JA, Strachan WE. Intracerebral hemorrhage due to ruptured venous aneurysm. Report of two cases. J Neurosurg 1978;49(5):739-743

4 Kelly KJ, Rockwell BH, Raji MR, Altschuler EM, Martinez AJ. Isolated cerebral intraaxial varix. AJNR Am J Neuroradiol 1995;16(8): 1633-1635

5 Hong W, Zheng X, Wu Z, et al. Clinical features and surgical treatment of trigeminal neuralgia caused solely by venous compression. Acta Neurochir (Wien) 2011;153(5):1037-1042

6 Inoue T, Hirai $\mathrm{H}$, Shimizu T, et al. Trigeminal neuralgia due to venous angioma: usefulness of preoperative 3D-MR imaging for microvascular decompression [in Japanese]. No Shinkei Geka 2012;21(7):556-561

7 Dross P, Raji MR, Dastur KJ. Cerebral varix associated with a venous angioma. AJNR Am J Neuroradiol 1987;8(2):373-374

8 Uchino A, Hasuo K, Matsumoto S, Ikezaki K, Masuda K. Varix occurring with cerebral venous angioma: a case report and review of the literature. Neuroradiology 1995;37(1):29-31

9 Hoell T, Hohaus C, Beier A, Holzhausen HJ, Meisel HJ. Cortical venous aneurysm isolated cerebral varix. Interv Neuroradiol 2004; 10(2):161-165

10 Tanju S, Ustuner E, Deda H, Erden I. Cerebral varix simulating a meningioma: use of 3D magnetic resonance venography for diagnosis. Curr Probl Diagn Radiol 2006;35(6):258-260

11 Kin T, Oyama H, Kamada K, Aoki S, Ohtomo K, Saito N. Prediction of surgical view of neurovascular decompression using interactive computer graphics. Neurosurgery 2009;65(1):121-128; discussion $128-129$

12 Miller J, Acar F, Hamilton B, Burchiel K. Preoperative visualization of neurovascular anatomy in trigeminal neuralgia. J Neurosurg 2008;108(3):477-482

13 Inoue T, Hirai $\mathrm{H}$, Shimizu T, et al. Ocular neuromyotonia treated by microvascular decompression: usefulness of preoperative 3D imaging: case report. J Neurosurg 2012;117(6):1166-1169

14 Sindou M, Howeidy T, Acevedo G. Anatomical observations during microvascular decompression for idiopathic trigeminal neuralgia (with correlations between topography of pain and site of the neurovascular conflict). Prospective study in a series of 579 patients. Acta Neurochir (Wien) 2002;144(1):1-12; discussion 12-13

15 Cho DY, Chang CG, Wang YC, Wang FH, Shen CC, Yang DY. Repeat operations in failed microvascular decompression for trigeminal neuralgia. Neurosurgery 1994;35(4):665-669; discussion 669670

16 Lee SH, Levy EI, Scarrow AM, Kassam A, Jannetta PJ. Recurrent trigeminal neuralgia attributable to veins after microvascular decompression. Neurosurgery 2000;46(2):356-361; discussion $361-362$ 